\title{
Evaluation de la surveillance épidémiologique de la rougeole : Analyse de la base de données du district sanitaire
} de Koulikoro de 2012-2018

\section{Evaluation of the epidemiological surveillance of measles: Analysis of the Koulikoro health district database from 2012-2018}

Coulibaly Hamadou' ${ }^{1}$ Sangho Oumar²,3, Sogodogo Seydou4, Keyembé Ken ${ }^{5}$, Dakouo Hyacinthe ${ }^{1}$

\author{
${ }^{1}$ Centre de santé de référence de Koulikoro, Mali \\ 2Département d'Enseignement et de Recherche en Santé \\ Publique, FMOS, USTTB, Mali \\ ${ }^{3}$ DER des Sciences Biologiques et Médicales FAPH, USTTB, \\ Mali \\ ${ }^{4}$ Inspection de la Santé, Ministère de la Santé et des Affaires \\ Sociales \\ ${ }^{5}$ AFENET (African Field Epidemiology Network),
}

Auteur correspondance : Dr Hamadou COULIBALY, Médecin généraliste, Epidémiologiste de terrain niveau intermédiaire, chargé de recherche au centre de santé de référence de Koulikoro, Mali ; Tél: (00223) 76453870 / 65791174; E-mail : drahmadouc@gmail.com / drhamadou_ml@yahoo.fr

\section{RESUME}

Introduction: La rougeole est une maladie éruptive fébrile, due à un virus (Morbillivirus). L'absence de résultats d'analyse sur la rougeole dans le district, nous a conduit à l'analyse des données de surveillance épidémiologique de la rougeole de 2012 à 2018, les objectifs étaient de décrire les caractéristiques de la maladie en temps lieux et personnes et d'identifier les facteurs associés à la survenue de la rougeole. Matériel et méthodes: Nous avons conduit une étude transversale, à l'aide des fiches de notifications, le cahier de déclaration, la liste linéaire et les fiches du laboratoire de 2012 à 2018, saisie et analyse sur Epi Info 7.2. Résultats : Dans notre étude, nous avons retrouvé $n=48$ cas suspects notifiés de 2012 à 2018 dont $6 \%$ des cas positif $(\lg \mathrm{M}+)$, le sexe masculin était le plus représenté $n=27$ (56) $P=0,38$, les sujets âgés de 15 ans et plus ont représenté la totalité des cas positifs $(\operatorname{lgM}) \mathrm{n}=3$ (100) $P=0,0002$, les sujets (suspects et confirmés) $n=6$ n'étaient pas vaccinés contre la rougeole $P=0,0002$, les cas suspects et confirmés $n=31(64,5)$ provenaient de la ville de Koulikoro. Conclusion: L'analyse de la base, nous a permis de retrouver trois cas suspects et confirmés dans le district. Les hommes étaient plus concernés que les femmes. Tous les cas avaient au moins 15 ans et aucun n'était vacciné contre la rougeole. Cette situation nécessiterait un renforcement de la vaccination de routine et une étude sur une plus longue période pouvant s'intéresser aux facteurs déterminants.

Mots clés: Analyse, base de données, rougeole, Koulikoro, Mali

\footnotetext{
ABSTRACT

Introduction: Measles is a febrile eruptive disease caused by a virus (Morbillivirus). The absence of measles analysis results in the district, led us to the analysis of measles epidemiological surveillance data from 2012 to 2018 , the objectives were to describe the characteristics
}

of the disease in time and person and to identify the factors associated with the occurrence of measles. Material and methods: We conducted a crosssectional study, using the notification forms, the declaration book, the linear list and the laboratory files from 2012 to 2018, entered and analyzed on Epi Info 7.2. Results: In our study, we found $n=48$ suspected cases from 2012 to 2018, of which 6\% were positive (lgM +), the male sex was the most represented $n=27(56) P=$ 0.38 , the elderly were 15 years and over represented all positive cases (lgM) $n=3(100) P=0.0002$. Subjects (suspected and confirmed) $n=6$ were not vaccinated against measles $P=0.0002$, the suspected and confirmed cases $n=31$ (64.5) came from the city of Koulikoro. Conclusion: The analysis of the database, allowed us to find three suspected and confirmed cases in the district. Men were more concerned than women. All cases were at least 15 years old and none were vaccinated against measles. This suggest a reinforcement of routine immunization and a study over a longer period that may be help to identify the determinant factors.

Keywords: Analysis, database, measles, Koulikoro, Mali

\section{Introduction}

La rougeole est une maladie éruptive fébrile, due à un virus de la famille des Paramyxoviridae (Morbillivirus) (1). Dans le monde entre 2000 et 2016, environ 20,4 millions de décès ont été évité par la vaccination contre la rougeole (2), qui fait de ce vaccin le meilleur investissement dans le domaine de la santé publique (3). En Afrique plusieurs pays ont enregistré des flambées épidémiques de la rougeole en 2018, et c'est Madagascar qui a enregistré 120000 cas avec environ 1000 décès (4). Certains pays ont ainsi procédé à l'évaluation de leur système de surveillance (5-7). La vaccination est l'un des moyens sures pour protéger les personnes contre la maladie (8). A l'opposé la faible couverture vaccinale accroit le taux de morbidité et de mortalité liées aux maladies évitables par la vaccination chez les enfants de moins de cinq ans et les cinq à quatorze ans, qui est accentuée dans certaines zones par des situations de crises sécuritaires et la famine (8). En 2018, le Mali a enregistré 1634 cas suspects de rougeole parmi lesquels 1408 ont été prélevés avec 476 positifs (IgM) (9). L'Organisation Mondiale de la Santé (OMS) recommande l'amélioration de la couverture vaccinale, le renforcement des systèmes de surveillance, la formation des agents de santé, l'amélioration de la qualité des données (10). Au Mali les principaux résultats montrent une amélioration dans les différents domaines 
de la stratégie d'amélioration de la couverture vaccinale (9).

Dans la région de Koulikoro en 2018, il a été enregistré 334 cas suspects dont 298 cas prélevés et 95 confirmés (IgM) (9). II a été noté la survenue d'épidémie ayant fait l'objet de riposte dans trois districts sanitaires durant cette même année, notamment Kati (Bacoumana et Dogodouma), Kangaba (10 aires de santé) et Kalabancoro (Kabala, et malgré que la couverture vaccinale anti rougeoleuse était de $98 \%$ (9).

La couverture vaccinale du district sanitaire de Koulikoro en 2018, était de $77 \%$ (9) qui est très largement inférieur au norme attendue $(\geq 95 \%)$. Selon les résultats de notre étude descriptive de l'analyse de la base de données de surveillance épidémiologique de la rougeole de 2012 à 2018, nous avons retrouvé 48 cas suspects de rougeole, tous prélevés avec 3 cas positifs (IgM) (11), et la dernière épidémie déclarée avec riposte remonte en 2013. L'importance de la maladie, l'efficacité du vaccin et la faiblesse de la couverture vaccinale dans le district sanitaire de Koulikoro, l'absence de résultats d'analyse antérieure de la base ont motivé la présente étude dont l'objectif est l'évaluation de la surveillance épidémiologique de la rougeole à travers l'analyse de la base de données du district sanitaire de Koulikoro de 2012-2018. Le but est de contribuer à l'amélioration du système de surveillance et de contrôle de cette maladie.

\section{Matériel et méthode}

II s'agissait d'une étude transversale. Nous avons procédé à une analyse des données de surveillance de la rougeole de 2012 à 2018 au niveau du centre de santé de référence de Koulikoro. Ont été exclus de l'étude les cas chez qui l'âge, le sexe et la provenance n'étaient pas spécifiés. Nous avons utilisés les fiches de notification, le cahier de déclaration des maladies, la liste linéaire des cas, les fiches de monitorage au niveau du laboratoire du CSRéf. Nous avons constitué une base de données sur Epi info 7.2. Nous avons réalisé des analyses descriptives et bivariées. Le test statistique utilisé a été le test exact de Fisher avec un seuil de significativité fixé à $5 \%$.

\section{Résultats :}

De 2012 à 2018, le district sanitaire de Koulikoro a notifié 48 cas suspects de rougeole dont trois sont revenus positifs (IgM) soit une incidence cumulée de 6\%. Parmi ces 48 cas suspects déclarés, seuls 13 soit $27 \%$ des prélèvements ont eu un résultat de laboratoire disponible. La totalité des cas avait été notifiée par les structures publiques notamment les Centres de Santé Communautaire (CSCOM) et le Centre de Santé de Référence de Koulikoro (CSRéf). Sur les 21 CSCOM fonctionnels que compte le district sanitaire de Koulikoro, 10 ont notifié (Figure 1) durant la période, de 2012 à 2018, concernée par la collecte des données d'où une proportion de $47,6 \%$.

Dans le cadre de cette surveillance nous avons constaté une moyenne de 6,8 cas suspects par an, avec une plus forte notification en 2016 avec 18 cas suspects, dont le seuil épidémique fixé à 5 cas suspects dans un mois a été dépassé au mois de mars 2016 (figure 2). Le sexe masculin était le plus représenté parmi les cas suspects avec $56,25 \%$. Les enfants de 0 à 5 ans ont été les plus représentés, le mode était de 5 ans et l'étendu entre 1-30 ans (figure 3). Dans l'analyse bivariée, les hommes avaient 1,58 fois plus de susceptibilité d'être atteints par la rougeole que les filles, mais la relation n'était pas significative $(p=0,38)$ (tableau I).

\section{Discussion}

De 2012 à 2018, le district sanitaire de Koulikoro a notifié 48 cas suspects de rougeole dont trois sont revenus positifs (IgM) avec une incidence de 6\%. Ces cas étaient tous âgés de 15 ans et plus, contrairement à la majorité des résultats de revues littéraires dans lesquelles les enfants de moins de 5 ans étaient les plus touchés $(5,12,13)$. Cela pourrait être expliqué par la faible proportion de disponibilité des résultats de $27 \%(\mathrm{~N}=48)$ à partir du laboratoire National de référence, à une insuffisance dans la qualité de la vaccination en termes d'atteinte des objectifs. L'analyse montre que le sexe masculin était le plus représenté avec $56,25 \%$ ( $N=48$ ). La même tendance a été rapportée par l'étude réalisée par Mitiku B. et al. en Ethiopie avec $65 \%(\mathrm{~N}=150)$ mais contraire à l'étude réalisée par Barry $\mathrm{D}$. et al à Sikasso avec 58,3\% ( $\mathrm{N=36}$ ) de sexe féminin (14). Dans notre étude la majorité des cas provenaient des zones urbaines contrairement à l'étude réalisée Jasem J. et al. en Irak ou les zones rurales étaient les plus représentées (13).

L'absence de vaccination est un déterminant majeur de la rougeole. Dans notre étude, aucun des trois cas n'était vacciné. Ce résultat est comparable à celui de l'étude réalisée par Farra A. et al., sur les données de 20072015 en République Centre Africaine (RCA), qui trouve une relation significative entre l'absence de vaccination et la survenue de la rougeole (15). En République Démocratique du Congo (RDC), la vaccination était un facteur significativement protecteur contre la rougeole avec un Rapport de Cotes (RC) ajusté à $95 \%$ de 0,20 $(0,15-0,26)(6)$. Nous n'avons pas pu avoir d'explication à la survenu de la rougeole chez les 15 ans et plus. Cependant la faiblesse de la couverture vaccinale était de $77 \%$ largement en deçà de la norme admise (9), cela pourrait avoir contribué à leur survenue comme trouvé dans l'étude du RDC (6).

Limite de l'étude : la taille de l'échantillon était faible mais surtout, aucun cas de rougeole n'était vacciné. Cela a impacté les analyses statistiques du RC. Cette taille ne permet pas de bien conclure du point de vue statistique. En outre la moitié des CSCOM (11/21) ainsi que les structures privées n'ont fait aucune notification durant toute cette périodes. Ils sont considérés comme muets dans le cadre de la surveillance et impact la taille de l'échantillon mais aussi la qualité de cette surveillance. 


\section{Conclusion}

L'analyse de la base, nous a permis de retrouver 48 cas suspects dont trois confirmés dans le district sanitaire de Koulikoro. Les hommes étaient plus concernés. Tous les cas avaient au moins 15 ans et aucun n'était vacciné contre la rougeole. Ceci implique un renforcement de la vaccination de routine et une étude sur une plus longue période pouvant s'intéresser aux facteurs déterminants.

\section{Références}

1. Médecins sans frontières. Prise en charge d'une épidémie de rougeole, Guide pratique à l'usage des médecins, infirmiers, techniciens de laboratoire, auxiliaires de santé et logisticiens. 2013.

2. Dabbagh A. Progress Toward Regional Measles Elimination - Worldwide, 2000-2017. MMWR Morb Mortal Wkly Rep [Internet]. 2018 [cité 13 juill 2019];67. Disponible sur: https://www.cdc.gov/mmwr/volumes/67/wr/mm6747a6.ht $\mathrm{m}$

3. OMS | Nouvelles données de surveillance de la rougeole pour 2019 [Internet]. WHO. [cité 20 juin 2019]. Disponible sur:

http://www.who.int/immunization/newsroom/measlesdata-2019/fr

4. Debela MB, Kahsay AB, Mokonnon TM, Shifaw ZG. Evaluation of Measles Surveillance Systems in Afar Region, Ethiopia: A Descriptive Evaluative Study, 2017. 5. Ameh CA, Sufiyan MB, Jacob M, Waziri NE, Olayinka AT. Evaluation of the Measles Surveillance System in Kaduna State, Nigeria (2010-2012). Online J Public Health Inform. 2016;8(3):e206.

6. Doshi RH, Mukadi P, Shidi C, Mulumba A, Hoff NA, Gerber S, et al. Field evaluation of measles vaccine effectiveness among children in the Democratic Republic of Congo. Vaccine. 26 juin 2015;33(29):3407-14.

7. Masresha B, Katsande R, Luce R, Fall A, Shibeshi M, Weldegebriel G, et al. Performance of National Measles Case-Based Surveillance Systems in The WHO African Region. 2012 - 2016. J Immunol Sci. 2 août 2018;Suppl:130-4.

8. Ibrahim BS, Usman R, Mohammed Y, Datti Z, Okunromade $\mathrm{O}$, Abubakar AA, et al. Burden of measles in Nigeria: a five-year review of casebased surveillance data, 2012-2016. Pan Afr Med J [Internet]. 22 janv 2019 [cité 30 août 2019];32(Suppl 1). Disponible sur: https://www.ncbi.nlm.nih.gov/pmc/articles/PMC6445333/ 9. DNS Annuaire Système Local d'Information Sanitaire 2018 VF du 27 avril.pdf [Internet]. [cité 25 juin 2019]. Disponible sur:
http://www.sante.gov.ml/docs/AnnuaireSLIS2018VFdu27 avril.pdf

10. OMS. Elimination de la rougeole d'ici 2020 stratégies pour la région africaine. 2011.

11. DRS Koulikoro. Base de données de surveillance épidémiologique. 2018.

12. Debela M, Bayray A, Marama T, Shifaw Z. Evaluation of Measles Surveillance Systems in Afar Region, Ethiopia: A Descriptive Evaluative Study, 2017. Int J Biotech Trends Technol. 18 janv 2019;9.

13. Jasem J, Marof K, Nawar A, Monirul Islam KM. Epidemiological analysis of measles and evaluation of measles surveillance system performance in Iraq, 20052010. Int J Infect Dis IJID Off Publ Int Soc Infect Dis. mars 2012;16(3):e166-171.

14. Barry D, Traoré B. Evaluation du système de surveillance de la rougeole dans le district sanitaire de Sikasso. 2016 p. P25.

15. Farra A, Loumandet TN, Pagonendji M, Manirakiza A, Manengu C, Mbaïlao R, et al. Epidemiologic profile of measles in Central African Republic: A nine year survey, 2007-2015. PLOS ONE. 20 mars 2019;14(3):e0213735. 
Liste des tableaux et figures :

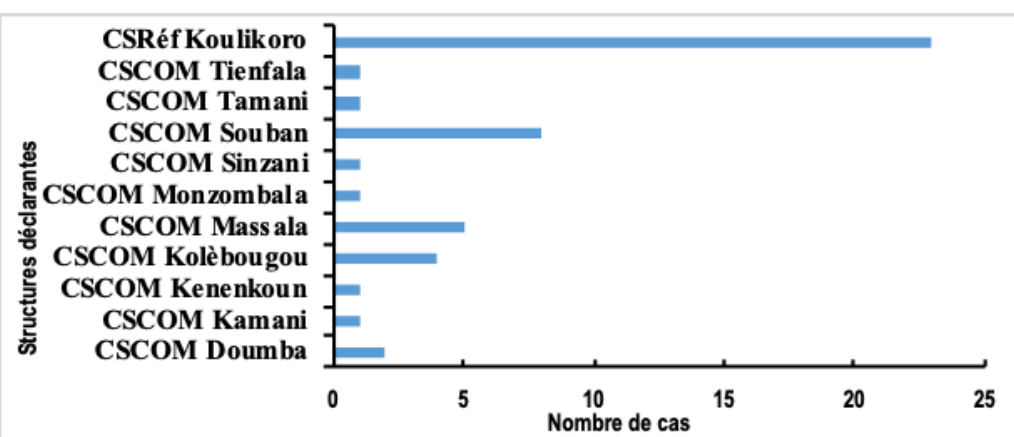

Figure 1 : Nombre de cas suspects de rougeole notifiés, par structure, district sanitaire de Koulikoro 2012-2018

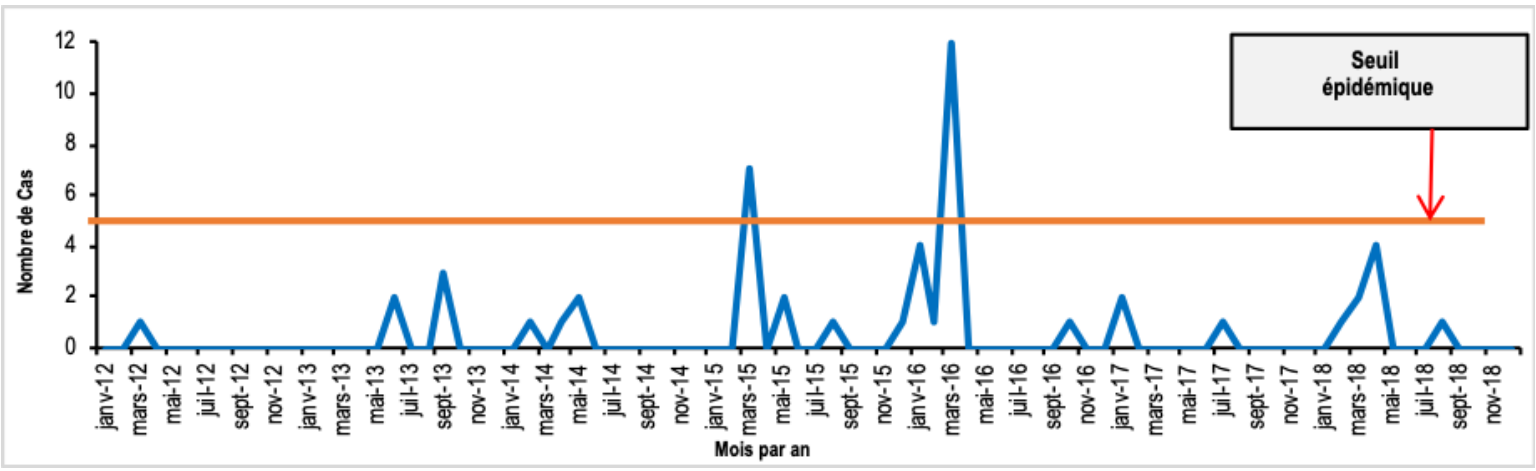

Figure 2 : Tendance des cas suspects de rougeole par mois et année, district sanitaire de Koulikoro, 2012 - 2018

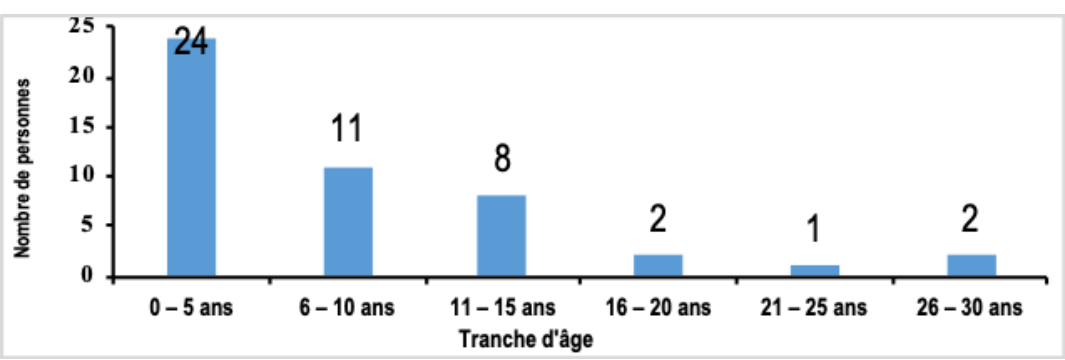

Figure 3 : Répartition des cas suspects de rougeole par tranche d'âge, district sanitaire de Koulikoro, 2012-2018

Tableau I : relation entre les facteurs, sexe, statut vaccinal, résidence, tranche d'âge et la rougeole dans le district sanitaire de Koulikoro, 2012-2018

\begin{tabular}{lcccc}
\hline \multicolumn{1}{c}{ Variables } & Rougeole Oui $(\mathrm{N}=3)$ & Rougeole Non (N=45) & RC [IC95\%] & $\mathbf{P}$ \\
\hline Sexe & 2 & 25 & $1,58[0,11-49,42]$ & 0,38 \\
Masculin & 1 & 20 & & \\
$\quad$ Féminin & 0 & 17 & $0[0,0-3,10]$ & 0,12 \\
$\begin{array}{l}\text { Statut vaccinal } \\
\text { Vacciné }\end{array}$ & 3 & 28 & & \\
Non vacciné & 2 & 29 & $1,10[0,07-34,48]$ & 0,48 \\
Résidence & 1 & 16 & & \\
Urbaine & & & & \\
Rurale & 0 & 43 & $0,0[0,0-0,14]$ & 0,0002 \\
Tranche d'âge & 3 & 2 & & \\
Moins de 15 ans & 0 & & & \\
15 ans et Plus & 3 & & & \\
\hline
\end{tabular}

